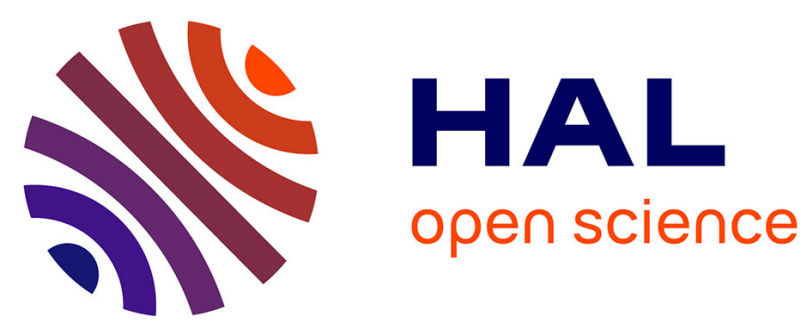

\title{
Revisited model-free controller for 3D autonomous navigation of quadrotor: Features analysis in harsh flight conditions
}

Yasser Bouzid, Houria Siguerdidjane, Yasmina Bestaoui

\section{- To cite this version:}

Yasser Bouzid, Houria Siguerdidjane, Yasmina Bestaoui. Revisited model-free controller for 3D autonomous navigation of quadrotor: Features analysis in harsh flight conditions. 13th IEEE International Conference on Control and Automation (ICCA 2017), Jul 2017, Ohrid, Macedonia. pp.915-920, 10.1109/ICCA.2017.8003183 . hal-01625851

\section{HAL Id: hal-01625851 \\ https://hal.science/hal-01625851}

Submitted on 27 Mar 2020

HAL is a multi-disciplinary open access archive for the deposit and dissemination of scientific research documents, whether they are published or not. The documents may come from teaching and research institutions in France or abroad, or from public or private research centers.
L'archive ouverte pluridisciplinaire HAL, est destinée au dépôt et à la diffusion de documents scientifiques de niveau recherche, publiés ou non, émanant des établissements d'enseignement et de recherche français ou étrangers, des laboratoires publics ou privés. 


\title{
Revisited model-free controller for 3D autonomous navigation of quadrotor: Features analysis in harsh flight conditions
}

\author{
Y. Bouzid, H. Siguerdidjane, Y. Bestaoui
}

\begin{abstract}
The performance in any flight system depends on the feedback control scheme. Therefore, in a first stage, we apply as a basis the so-called Model Free Control (MFC) for the development of nonlinear flight control law that could be implemented without much difficulty. As second step, an indepth discussion with respect to the control performance is highlighted. Due to the novel form that we propose, we can raise the performance and the robustness level with regards to structured or unstructured uncertainties. Thus, this nonlinear control that we call Revisited-MFC (R-MFC) is compared with other strategies and in particular with the use of the basic MFC. A detailed evaluation in terms of consumed energy, accuracy and robustness by considering several scenarios and using several metrics is presented. The numerical simulations have shown satisfactory results through an application to a small Vertical Take-Off and Landing (VTOL) quadrotor.
\end{abstract}

\section{INTRODUCTION}

In comparison with the traditional helicopters, the quadrotor is considered as a good case study to design, to analyze and to implement flight control strategies. Parallel to this, its widespread applications in numerous missions including military, governmental and academic missions motivate researchers in aeronautics [1] and robotics [2] to investigate and assess control techniques, which are tremendously required for the autopilot achievements.

It is necessary to design a controller such that the quadrotor will be able to follow a predefined trajectory, particularly in the presence of disturbances. For this reason, many studies have led to the development of sophisticated nonlinear control laws (as for instance [3-8]). For the sake of significant improvement, we seek to develop alternative methodologies to manage the system while maneuvering in harsh environments. The objectives of control may be focused on the robustness (w.r.t. external disturbances, unmodeled dynamics, parametric uncertainties, noise...), behavior (e.g. rapidity, accuracy, damped response...), consumed energy, etc.

In this regards, we are interested in exploring other theories that are not up to day sufficiently explored for the design of quadrotors flight controllers. In some recent papers, Model Free Control (MFC) has been applied to quadrotors, as for instance [9-10] where a continuous updating of the inputoutput of a very-local model is involved. In fact, the control of a system with a free model has been used, since many decades, on the basis of fuzzy logic control or the more popular one for linear systems through Ziegler Nichols method [11]. So the actual MFC looks like the solution using

Y. Bouzid is with IBISC laboratory, Université Paris-Saclay, Evry, France ( e-mail: yasseremp@gmail.com)

H. Siguerdidjane is with L2S, CentraleSupélec, Université Paris-Saclay, Gif sur yvette, France (e-mails:Houria.Siguerdidjane@centralesupelec.fr).

Y. Bestaoui is with IBISC laboratory, Université Paris-Saclay, Evry, France (Yasmina.Bestaoui@ufrst.univ-evry.fr) a kind of Ziegler Nichols method sample by sample, minimizing the tracking errors at each sample through the estimation of some quantities and linked to the linearization by feedback principle and the calculation of the PI or PID controllers. So, its use as basis of control will allow to compensate the uncertainties as well as other disturbances. This is due to the anticipation nature of the unmodeled dynamics and system uncertainties, which makes the control possible even with disturbances. It is employed in many applications such as: planar manipulator [12] as well as mobile robot [13], etc.

Throughout this paper, a performance assessment is presented via results of several illustrations, scenarios and numerical simulations, with complementary comments of the proposed revisited strategy of control with respect to other techniques. Particular attention is paid to the tracking accuracy and energy consumption of each control strategy considering some performance criteria, such as Maximum Absolute Error (MAE), Integral Squared Error (ISE) and Integral Squared Control Input (ISCI).

This paper is structured as follows. The second section concerns the dynamics of the VTOL quadrotor and the control architecture. Section 3 introduces the design of the nonlinear control approaches. In Section 4, the results from numerical simulations test the effectiveness of the proposed control strategies under different operating conditions for a quadrotor. The final section gives some conclusions.

\section{VEHICLE DYNAMICS BACKGROUND \& CONTROL ARCHITECTURE}

The X-shaped quadrotor has four rotors with twin-bladed propellers. As shown in Figure 1, the system operates in two coordinate frames: the inertial fixed frame $R_{0}\left(O_{0}, X, Y, Z\right)$ and the body frame $R_{1}\left(O_{1}, X_{1}, Y_{1}, Z_{1}\right)$.

Let $\eta=(\varphi, \theta, \Psi)^{T}$ describes the orientation of the aerial vehicle (Roll, Pitch, Yaw) and $\chi=(x, y, z)^{T}$ denotes its absolute position. Frequently, Euler-Lagrange formalism or Newton-Euler formalism are considered for the modeling of quadrotors where the general dynamic model has been presented in a number of papers (see [4,14-16]) and will not be discussed here again.

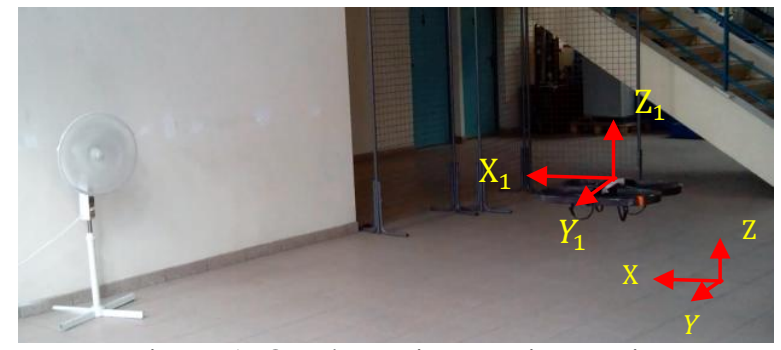

Figure 1. Quadrotor in experimentations. 
In conditions of flying at low indoor speed or hovering [17], the simplified dynamic model, in terms of inertial position $\chi$ and rotation $\eta$, may be written as:

$$
\ddot{\xi}=\left[\begin{array}{c}
\frac{c_{\Psi} s_{\theta} c_{\varphi}+s_{\Psi} s_{\varphi}}{m} u_{z} \\
\frac{s \Psi s_{\theta} c_{\varphi}-c_{\Psi} s_{\varphi}}{m} u_{z} \\
-g+\frac{c_{\theta} c_{\varphi}}{m} u_{z} \\
\dot{\theta} \dot{\Psi}\left(\frac{I_{y}-I_{z}}{I_{x}}\right)+\frac{u_{\varphi}}{I_{x}} \\
\dot{\varphi} \dot{\Psi}\left(\frac{I_{z}-I_{x}}{I_{y}}\right)+\frac{u_{\theta}}{I_{y}} \\
\dot{\varphi} \dot{\theta}\left(\frac{I_{x}-I_{y}}{I_{z}}\right)+\frac{u_{\Psi}}{I_{z}}
\end{array}\right]
$$

where $s_{(.)}$and $c_{(.)}$are abbreviations for $\sin ($.$) and \cos ($. respectively, $m$ is the mass, $g$ is the gravity acceleration and $u_{z}$ is the total of thrusts. $I=\operatorname{diag}\left(I_{x}, I_{y}, I_{z}\right)$ is the diagonal inertia matrix and $\tau=\left(u_{\varphi}, u_{\theta}, u_{\Psi}\right)^{T}$ is the control torque vector. $\xi=(\chi, \eta)^{T}$ denotes the output vector.

The quadrotor has four inputs and six degrees of freedom. So, in order to render the system fully-actuated and to simplify the design of the controller, two virtual inputs $u_{x}$ and $u_{y}$ are considered

$\left\{u_{x}=c_{\psi} s_{\theta} c_{\varphi}+s_{\Psi} s_{\varphi}\right.$

$\left\{u_{y}=s_{\Psi} s_{\theta} c_{\varphi}-c_{\Psi} s_{\varphi}\right.$

where the following equality must be verified

$u_{x}^{2}+u_{y}^{2}+c_{\theta}^{2} c_{\varphi}^{2}=1$

From system (2), we accept that

$\left\{\begin{array}{c}\varphi_{r}=\arcsin \left(u_{x} \sin \Psi_{r}-u_{y} \cos \Psi_{r}\right) \\ \theta_{r}=\arcsin \left(\frac{u_{x} \cos \Psi_{r}+u_{y} \sin \Psi_{r}}{\cos \varphi_{r}}\right)\end{array}\right.$

System (4) delivers the required reference trajectories for the rotation sub-system.

Considering the virtual inputs (2), clearly, the Multi-Input Multi-Output system may be divided into a set of SingleInput Single-Output (SISO) systems affine in the control written as

$\ddot{q}=f_{q}(q, \eta)+\left.g_{q}(\eta, w) u_{q}\right|_{q=\{x, y, z, \varphi, \theta, \Psi\}}$

where $f_{q}(),. g_{q}($.$) can be identified from the overall$ dynamic model (1). $w$ may be considered as a known time varying term. The system parameters are provided in Table 1.

Table 1. Quadrotor parameters.

\begin{tabular}{|c|c|c|c|}
\hline$m(\mathrm{~kg})$ & 0.429 & $I_{y}\left(\mathrm{~kg} \cdot \mathrm{m}^{2}\right)$ & 0.0029 \\
\hline$I_{x}\left(\mathrm{~kg} \cdot \mathrm{m}^{2}\right)$ & 0.0022 & $I_{z}\left(\mathrm{~kg} \cdot \mathrm{m}^{2}\right)$ & 0.0048 \\
\hline
\end{tabular}

\section{R-MFC FLIGHT CONTROLLER DESIGN}

We consider a class of nonlinear SISO systems for $t \in[0, \infty)$ given by

$y^{(n)}(t)=f\left(\zeta, w_{f}\right)+g\left(\zeta, w_{g}\right) u(t)$

where $\zeta=\left(y(t), \dot{y}(t) \ldots, y^{(n-1)}(t)\right)^{\mathrm{T}} \in D_{\zeta} \subset \mathbb{R}^{n}$ is an $n$ dimentional state vector, $u \in \mathcal{U} \subset \mathbb{R}$ is a scalar input, $y \in$ $D_{y} \subset \mathbb{R}$ is a scalar output, $f():. D_{\zeta} \rightarrow \mathbb{R}$ is a multi-variable nonlinear function. $g():. D_{\zeta} \rightarrow \mathbb{R}$ is an input nonlinear function with $g() \neq$.0 in domain $D_{\zeta} \cdot w_{g}$ and $w_{f}$ are time varying known terms.

In the literature, the use of MFC is linked to the linear PID or PD controller under the name intelligent-PID ( $i$ PID) and $i \mathrm{PD}$ for $n=2$ or $n=1$ respectively. The early versions of the iPID may be found in [11]. However, in [18], it is shown that the $i$ PID brings no better performance than the classical PID. Moreover, the existing MFC techniques consider only the input-output signals in order to design the controller. However, we have, currently, a nominal model for the controlled system even if it is an approximated version that can be exploited to synthesize the controller. Therefore, for a significant improvement, in this paper, we propose to use the MFC with a nonlinear feedback technique rather than using it with the classical linear controllers (PI or PID) by considering the available nominal model where the MFC principle is investigated for the unknown dynamics of the system that can represent: bounded external disturbances, neglected dynamics...So, a revisited MFC (R-MFC) is proposed.

The input-output relationship of the anticipated disturbed model may be represented by the form

$y^{(v)}=f\left(\zeta, w_{f}\right)+g\left(\zeta, w_{g}\right) u+\Delta(\zeta, u)$

Motivated by the system model (6), the mismatch term could be written as:

$\Delta(\zeta, u)=\tilde{f}(\zeta)+\kappa u$

where $v$ is the order of the anticipated model, $\kappa$ is an input scaling factor and $\tilde{f}(\zeta)$ is the disturbance dynamics.

From equations (7) and (8), it comes that $y^{(v)}=f\left(\zeta, w_{f}\right)+\left(g\left(\zeta, w_{g}\right)+\kappa\right) u+\tilde{f}(\zeta)$

According to the MFC strategy, the estimated term $\tilde{f}(\zeta)$ is continuously updated. From (9), we define

$\tilde{f}(\zeta)=\hat{y}^{(v)}-\left(g\left(\zeta, w_{g}\right)+\kappa\right) \hat{u}-f\left(\zeta, w_{f}\right)$

Using the knowledge of the past input $\hat{u}$ and $\kappa$ where $\hat{y}^{(v)}$ denotes the $v^{\text {th }}$ derivative of the measure $y$ in the previous time interval, the value of $\tilde{f}(\zeta)$ is estimated. This estimation is of course valid for a short period of time and it should be continuously updated on every iteration of the closed loop controller.

This updated value injects the required change in the control input where the control input for R-MFC strategy is written as follows

$u=\frac{-\tilde{f}(\zeta)+u_{f}}{g\left(\zeta, w_{g}\right)+\kappa}$

where $u_{f}$ is an auxiliary input. Substituting (11) into (9) yields

$y^{(v)}=f\left(\zeta, w_{f}\right)+u_{f}$

$u_{f}$ should be selected in order to push the tracking error of system (12) toward the origin. The direct mission is to investigate the controller $u_{f}$ that implies the dynamic model inverse of class of nonlinear affine SISO system (12) and occurs in closed loop control architecture.

The inversion of the nonlinear model requires the relationship between the input $u_{f}$ and the output $y$. A broad range of techniques already published investigating this classic problem. In our previous work [19], we proposed to employ the flatness property to perform the inverse of nonlinear system even with non minimum phase systems. Herein, for this particular case, we obtain $u_{f}=y^{(v)}-f\left(\zeta, w_{f}\right)$

We show that if we change, in a new manner, the dynamic inversion based control (13) in order to get a closed loop structure; we can raise the performance and the 
robustness level with regards to structured or unstructured uncertainties. We will address in the following, a simple analytic formulation for the design of our controller, synthesized for nonlinear Single-Input, Single-Output (SISO) systems, which involves a closed loop control structure.

Let $\mu$ denotes some lower bounded positive constant. $\forall n \geq 1$, assume that the tracking error $\left(e=y_{r}-y\right)$ has a first order dynamic

$\dot{e}+\frac{1}{\mu} e=0$

From equation (14), we get $\dot{e}=-\frac{1}{\mu} e$.

For a short period of time, the reference trajectory is considered constant, which allows writing

$y^{(1)}=\frac{e}{\mu}$

and the successive time derivatives are

$y^{(i)}=\frac{e^{(i-1)}}{\mu} i=2, \ldots, m$

where $m=\max \{n-1, v\}$

The main idea here is to change the form of equation (15) and write it under a general form involving all the tracking error time derivatives $\left(e^{(i-1)}, \ldots, e\right)$. Introducing two parameters, $\left(\alpha_{1}, \beta_{1}\right) \in \mathbb{R} \times \mathbb{R}$ verifying $\alpha_{1}+\beta_{1}=1$, we obtain

$y^{(i)}=\frac{\alpha_{1}}{\mu} e^{(i-1)}+\frac{\beta_{1}}{\mu}\left(y_{r}-y\right)^{(i-1)} i=2, \ldots, m$

Then

$y^{(i)}=\frac{\alpha_{1}}{\mu} e^{(i-1)}-\frac{\beta_{1}}{\mu} y^{(i-1)} \quad i=2, \ldots, m$

By recurrence, we obtain a general form

$\begin{aligned} y^{(i)} & =\sum_{k=1}^{k=i}(-1)^{k+1} \frac{\alpha_{(k)} \prod_{j=1}^{k} \beta_{(j-1)}}{\mu^{k}} e^{(i-k)} \\ i & =2, \ldots, m\end{aligned}$

with the initial term $y^{(1)}=\frac{e}{\mu}$ and $\left(\alpha_{(k)}, \beta_{(k)}\right) \in \mathbb{R} \times \mathbb{R}$ verifying $\alpha_{(k)}+\beta_{(k)}=1, \alpha_{(i)}=1$ and $\beta_{(0)}=1$.

Our objective is to design one controller that drives system (12) output to track a desired trajectory. Let us write again controller (13) in terms of the tracking error by using relationship (18).

$u_{f}=\sum_{k=1}^{k=v}(-1)^{k+1} \frac{\alpha_{(k)} \prod_{j=1}^{k} \beta_{(j-1)}}{\mu^{k}} e^{(v-k)}-$

$f\left(\left.\sum_{k=1}^{k=i}(-1)^{k+1} \frac{\alpha_{(k)} \prod_{j=1}^{k} \beta_{(j-1)}}{\mu^{k}} e^{(i-k)}\right|_{i=2, \ldots, n-1}, w_{f}\right)$

Also, this auxiliary input is of course valid for a short period of time and it should be continuously updated on every iteration of the closed loop controller. Figure 3 clarifies this approach.

The R-MFC presents several benefits such as the simplicity of its structure. Moreover, it exhibits a good level of robustness with self adaption in case of uncertainties. However, the use of classical PID may reduce the capabilities of the promising features of the controller. Also, the classic dynamic inversion technique involves the cancelation of the nonlinearities. Thus, if the model is inaccurate or contains some varying variables that are highly influenced by the environment changes (temperature, pressure, etc.), this cancelation may be not exact and engenders other equilibrium points of the closed loop system or causes the instability. This drawback is tackled by our proposed controller, where the plant-model mismatch is canceled by using the tracking error in the inversion process that penalizes the deviations between the output and its set point. Therefore, the proposed controller boosts the robustness capabilities level of the system.

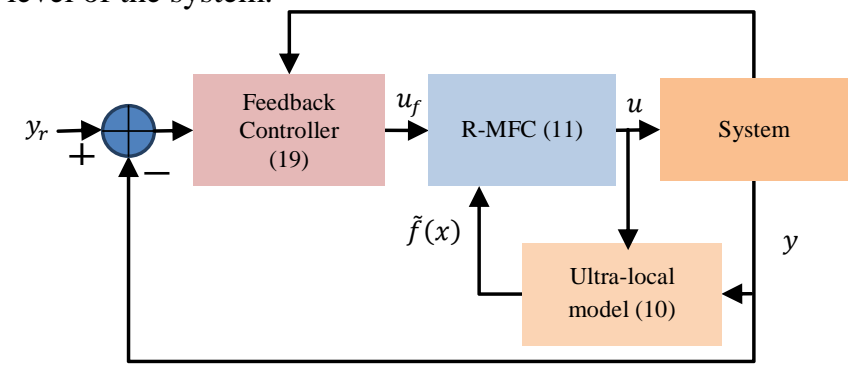

Figure 2. R-MFC scheme.

\section{RESULTS AND DISCUSSION}

For a significant analysis of the features of the proposed controller, three additional nonlinear controllers are considered for the sake of comparison. The first one is recently published by the authors that is the Nonlinear Internal Model Control (NLIMC) and exhibits very promising results (for details one may refer to [19]) whilst the second one is traditionally applied for quadrotors i.e. BackStepping approach (BS). The third one concerns the classical form of MFC applied to the quadrotor by Wang [9]. The control parameters, depicted in Table 2, are tuned by optimizing the integral square error

$I S E=\frac{1}{t_{f}-t_{i}} \int_{t_{i}}^{t_{f}}\left(\xi_{r}-\xi\right)^{T}\left(\xi_{r}-\xi\right) d t$

where $t_{i}$ and $t_{f}$ denote the initial and the final instants of optimization respectively. The optimization is done, via genetic algorithms (GA), in the ideal case then kept for the entire proposed scenarios.

Table 2: control parameters.

\begin{tabular}{|c|c|c|c|c|c|}
\hline$\mu_{x}$ & 0.08 & $\alpha_{x}$ & 3.18 & $\kappa_{x}$ & 0.5 \\
\hline$\mu_{y}$ & 0.03 & $\alpha_{y}$ & 1.08 & $\kappa_{y}$ & 0.5 \\
\hline$\mu_{z}$ & 0.12 & $\alpha_{z}$ & 4.78 & $\kappa_{z}$ & 0.5 \\
\hline$\mu_{\varphi}$ & 0.09 & $\alpha_{\varphi}$ & 1.80 & $\kappa_{\varphi}$ & 0.5 \\
\hline$\mu_{\theta}$ & 0.26 & $\alpha_{\theta}$ & 1.93 & $\kappa_{\theta}$ & 0.5 \\
\hline$\mu_{\Psi}$ & 0.04 & $\alpha_{\Psi}$ & 1.27 & $\kappa_{\Psi}$ & 0.5 \\
\hline
\end{tabular}

The overall system dynamics and control laws are implemented on Matlab program. The total simulation time is 40 seconds and the sampling time is 0.01 seconds. We simulate the behavior of the quadrotor using the system available parameters (see Table 1).

The effectiveness of the controller is investigated not only in the ideal case but also in the presence of different disturbances namely: model parameters uncertainties, extra payload, sensors noise and gust of wind following the same protocol and respecting the same control conditions. These scenarios are:

- Basic scenario: In this scenario, after the take-off, the quadrotor tracks a square reference trajectory of width $L_{r}=2 m$ (see Figure 3 ). When it arrives at each corner, the quadrotor hovers for a few seconds then it flies to the next corner where the flight duration between two corners is fixed at $T=5$ seconds. Therefore, this trajectory may be described as 
$\sigma(t)= \begin{cases}0 & \text { when } 0 \leq t \leq t_{1} \\ L_{r} \frac{\left(t-t_{1}\right)^{5}}{\left(t-t_{1}\right)^{5}+\left(T-t+t_{1}\right)} & \text { when } t_{1}<t \leq t_{2} \\ L_{r} & \text { when } t_{2}<t \leq t_{3} \\ L_{r}-L_{r} \frac{\left(t-t_{3}\right)^{5}}{\left(t-t_{3}\right)^{5}+\left(T-t+t_{3}\right)} & \text { when } t_{3}<t \leq t_{4} \\ 0 & \text { when } t_{4}<t \leq t_{f}\end{cases}$

with $T=5$ seconds, $t_{f}=40$ seconds and $L_{r}=2$ meters.

$x_{r}=\sigma(t)$ with $t_{1}=5, t_{2}=t_{1}+T, t_{3}=25, t_{4}=t_{3}+T$

$y_{r}=\sigma(t)$ with $t_{1}=10, t_{2}=t_{1}+T, t_{3}=30, t_{4}=t_{3}+T$

$z_{r}=\sigma(t)$ with $t_{1}=0, t_{2}=t_{1}+T, t_{3}=35, t_{4}=t_{3}+T$

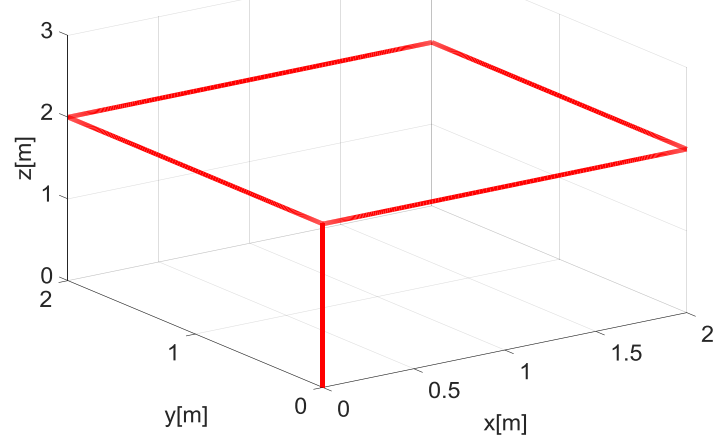

Figure 3. 3D square reference trajectory.

- Parameters uncertainties: In this scenario, some random parameter uncertainties are given. Supposing the inertia matrix elements and the aerodynamic coefficients are underrated $50 \%$ of the real values.

- Extra payload: In this scenario, the quadrotor is supposed using a heavy camera with additional mass that represents $50 \%$ from the initial mass of quadrotor. The camera is supposed as rigid compact body located at the center of mass of quadrotor.

- Sensor noise: In this scenario, we test the tolerance of controllers to noise that may affect the measured signals. Thus, we add the sensor noise on the states of the system. The expression of the noisy states is

$\left\{\tilde{\chi}=\chi+N_{c} \operatorname{rand}()\right.$.

$\left\{\tilde{\eta}=\eta+N_{c} \operatorname{rand}()\right.$.

The function $\operatorname{rand}($.$) is a Matlab function, which$ generate a random number between 0 and $1 . N_{c}$ is a scale parameter to adjust the level of noise.

- Wind disturbance: This scenario is dedicated to test the stability of control systems while encountering environment disturbances, namely gust of wind. Here, we assume that the wind causes the same acceleration intensity on all $X, Y, Z$-axes. These accelerations are considered as perturbations added to the equations related to the forces in the quadrotor model. Therefore, the disturbed model is expressed as follows

$\ddot{\tilde{x}}=\ddot{x}+a_{x}(t)$

$\ddot{\tilde{y}}=\ddot{y}+a_{y}(t)$

$\ddot{\tilde{z}}=\ddot{z}+a_{z}(t)$

The profile of this acceleration is depicted in Figure 4.

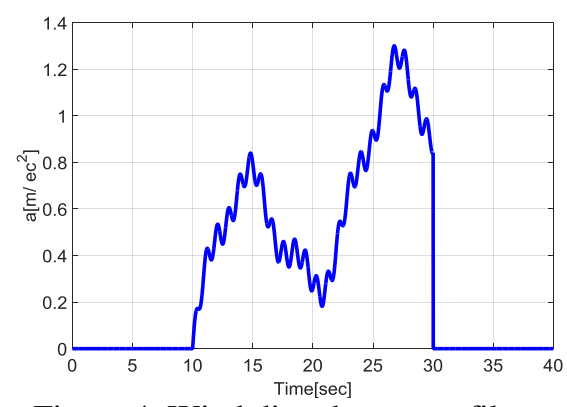

Figure 4. Wind disturbance profile.

We firstly implement the controllers for the basic scenario. We plot separately, the attitude angles in Figure 5, the tracking errors of the translations along $\mathrm{X}, \mathrm{Y}, \mathrm{Z}$-axes in Figure 6, the global thrust in Figure 7 and the regain control inputs in Figure 8.

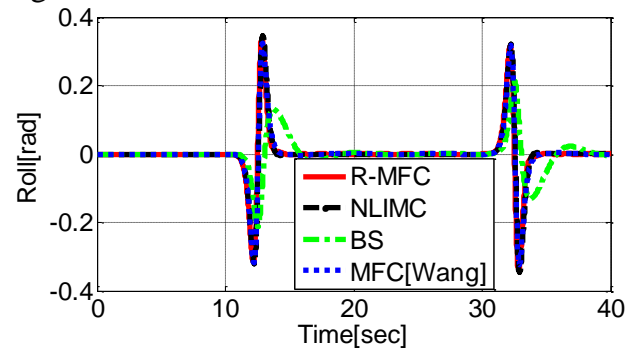

(a)

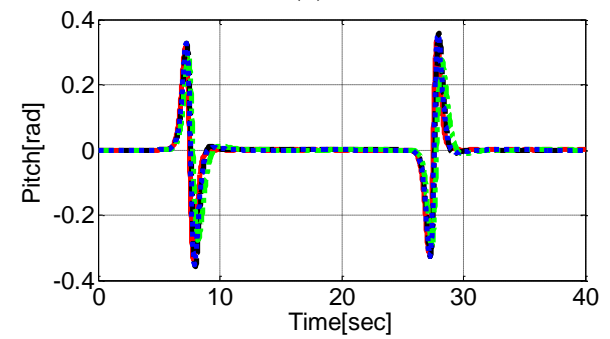

(b)

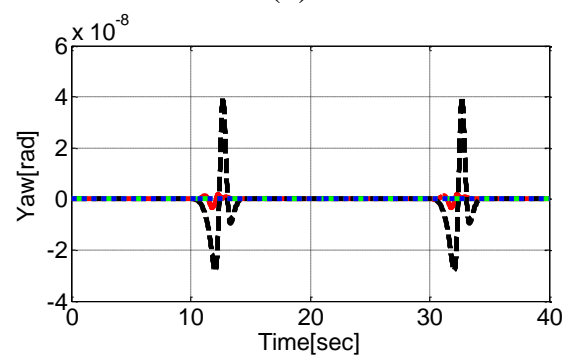

(c)

Figure 5. Attitude angles.

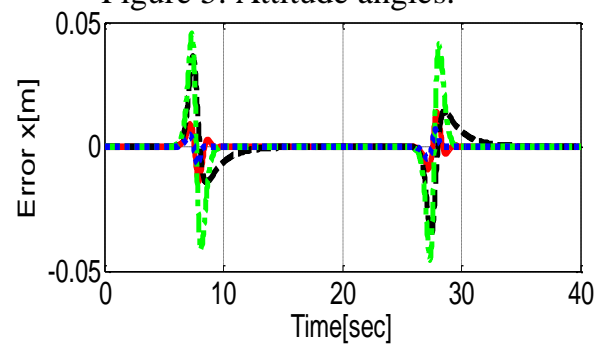

(a) 


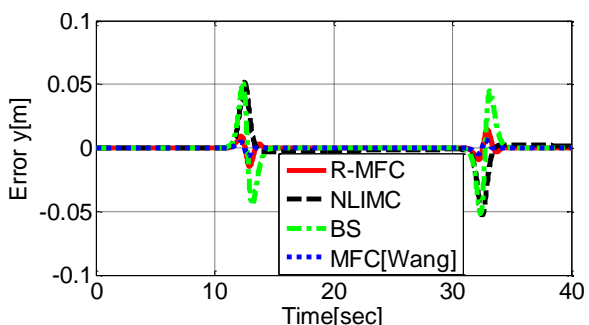

(b)

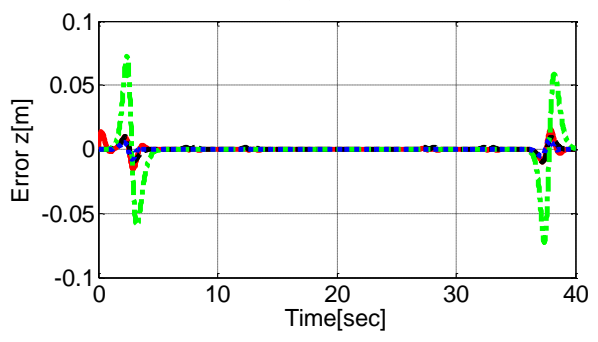

(c)

Figure 6. Tracking errors.

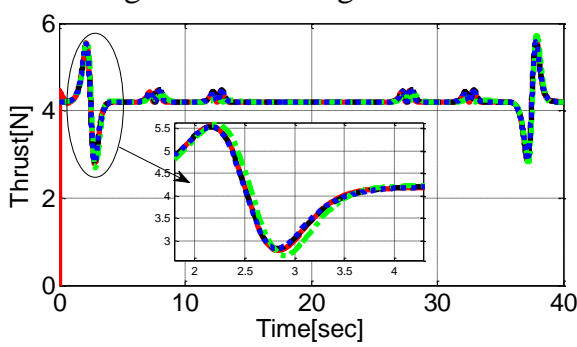

Figure 7. Global thrust.

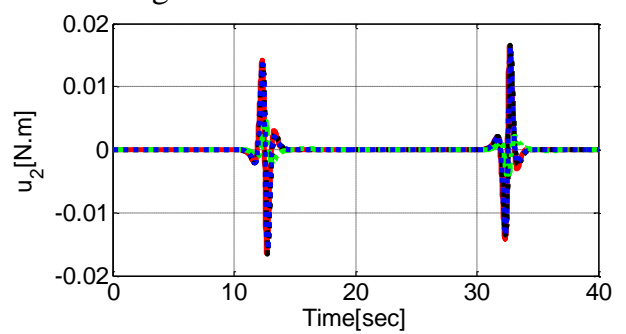

(a)

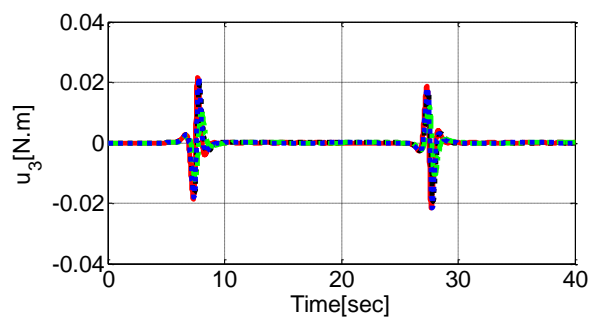

(b)

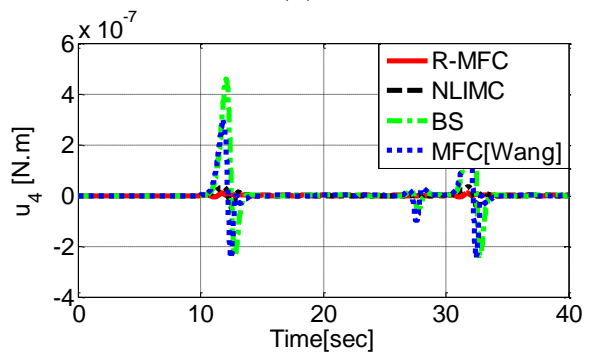

(c)

Figure 8. Control inputs.
From the basic scenario displayed in Figure 5-Figure 8, the quadrotor follows its reference trajectory in good manner and satisfactory accuracy. The consumed energy is almost the same for the three controllers. Clearly, the R-MFC exhibits a damped response for which the overshoot is the smallest (see Figure 6).

Obviously, the previous curves are not able to show the differences between the controllers the fact that all the techniques exhibit acceptable results in the ideal case. Therefore, we suggest quantifying the obtained results in order to get a close view on the features for a deep analysis of the performance. Therefore, some statistical analysis metrics are considered, such as the integral square error $I S E=$ $\int_{t_{0}}^{t_{f}}\left(e_{x}^{2}(t)+e_{y}^{2}(t)+e_{z}^{2}(t)\right) d t$, the maximum absolute error $M A E=\max \left(\left|e_{x}\right|,\left|e_{y}\right|,\left|e_{z}\right|\right)$ and the integral square control input (ISCI $\left.=\int_{t_{0}}^{t_{f}} u_{1}(t) d t\right)$. The obtained values for the remaining scenarios are depicted in Table 3.

Table 3: Metrics values.

\begin{tabular}{|c|c|c|c|c|}
\hline & $\mathrm{BS}$ & NLIMC & MFC [9] & R-MFC \\
\hline \multicolumn{5}{|c|}{ Basic scenario } \\
\hline ISE & 0.0152 & 0.003 & 0.002 & 0.002 \\
\hline MAE & 0.072 & 0.052 & 0.008 & 0.015 \\
\hline ISCI & 716.031 & 710.537 & 718.012 & 717.855 \\
\hline \multicolumn{5}{|c|}{ Parameters uncertainties } \\
\hline ISE & 2.972 & 0.043 & 1.18 & 0.002 \\
\hline MAE & 0.411 & 0.051 & 0.250 & 0.015 \\
\hline ISCI & 713.141 & 712.722 & 719.727 & 718.133 \\
\hline \multicolumn{5}{|c|}{ Extra payload } \\
\hline ISE & 0.767 & 0.021 & 0.297 & 0.003 \\
\hline MAE & 0.242 & 0.079 & 0.125 & 0.021 \\
\hline ISCI & 1612.613 & 1616.945 & 1619.68 & 1615.730 \\
\hline \multicolumn{5}{|c|}{ Sensors noise } \\
\hline ISE & 0.047 & 0.004 & 0.006 & 0.017 \\
\hline MAE & 0.093 & 0.051 & 0.0147 & 0.026 \\
\hline ISCI & 719.277 & 716.356 & 717.894 & 717.900 \\
\hline \multicolumn{5}{|c|}{ Wing gust } \\
\hline ISE & 0.028 & 0.136 & 0.009 & 0.002 \\
\hline MAE & 0.072 & 0.140 & 0.024 & 0.015 \\
\hline ISCI & 675.040 & 675.150 & 676.790 & 676.590 \\
\hline
\end{tabular}

Overall, as illustrated by Table 3 , the three controllers exhibit an acceptable behavior regardless the external effect. Nevertheless, the extra payload as the gust of wind requests additional thrust in order to ensure good performance.

One may notice that, from Table 3 and for the basic scenario, the BS technique is the less accurate technique (ISE=0.0152) followed by the NLIMC technique (ISE=0.003) while the MFC and R-MFC are the most accurate techniques (ISE=0.002). We also observe that the MFC consumes almost more energy while the NLIMC consumes less energy.

The accuracy of control degrades less and more for the BS, NLIMC and the MFC techniques for each scenario. However, the same performance is almost ensured by the RMFC regardless the considered scenarios except for the noise scenario. It is worthwhile to note that the R-MFC is insensitive to the disturbances where the thrust is requested according to the disturbance intensity in order to keep the same performance. 
The MAE measures the level of overshoot in the system time response, which is not recommended for the quadrotors the fact that physical oscillations may occur during the flight. Therefore, Table 3 shows that the best controller is the RMFC.

Finally, among the considered approaches, R-MFC exhibits the best performance in terms of accuracy and damped response almost for the overall scenarios with acceptable level of energy consumption.

\section{CONCLUSION}

In this paper, a novel way to design a MFC controller, called R-MFC, is described. It uses an auxiliary input and by bringing some changes (see Section III), it operates in closed loop form. This controller differs from the one that can be derived by using classical PD or PID. It improves the performance with respect to structured and unstructured uncertainties. The strong efficiency of the approach that we have proposed here is demonstrated in multiple test scenarios. The settling time is shown to be quite fast with good accuracy and a high level of robustness is ensured with respect to parameters uncertainties or external disturbances. The autonomous vehicle, exhibits good performance under the wind gust and maintains its defined position very well.

Numerical simulations have been performed on the nonlinear dynamic model of quadrotor in order to test the effectiveness of the designed control systems. The efficiency of the proposed controller is compared with other nonlinear controllers by emphasizing better performance.

\section{REFERENCES}

[1] H. Huang, G. M. Hoffmann, S. L. Waslander, and C. J. Tomlin, "Aerodynamics and control of autonomous quadrotor helicopters in aggressive maneuvering," In 2009 IEEE International Conference on Robotics and Automation, 2009, pp. 3277-3282.

[2] P. Pounds, R. Mahony, and P. Corke, "Modelling and control of a large quadrotor robot," Control Engineering Practice, vol. 18, no. 7, pp. 691-699, Jul. 2010.

[3] A. A. Mian and W. Daobo, "Nonlinear Flight Control Strategy for an Underactuated Quadrotor Aerial Robot," in IEEE International Conference on Networking, Sensing and Control, 2008. ICNSC 2008, 2008, pp. 938-942.

[4] Y. Bouzid, H. Siguerdidjane, Y. Bestaoui, and M. Zareb, "Energy Based 3D Autopilot for VTOL UAV under Guidance \& Navigation Constraints," Journal of Intelligent \& Robotic Systems, pp. 1-22, 2016. Doi: 10.1007/s10846016-0441-1.

[5] P. Castillo, R. Lozano, and A. E. Dzul, Modelling and Control of Mini-Flying Machines. Springer Science \& Business Media, 2005.

[6] Y. Bouzid, H. Siguerdidjane and Y. Bestaoui, "Real time Autopilot based on Immersion \& Invariance for Autonomous Aerial Vehicle", 20th IFAC Symposium on Automatic Control in Aerospace (ACA), IFAC-PapersOnLine, vol. 49, no. 17, pp. 176-181, Sherbrooke, Quebec, Canada 2125August, 2016.

[7] G. M. Hoffmann, H. Huang, S. L. Waslander, and C. J. Tomlin, "Precision flight control for a multi-vehicle quadrotor helicopter testbed," Control Engineering Practice, vol. 19, no. 9, pp. 1023-1036, Sep. 2011.

[8] P. Pounds (2008) Design, construction and control of a large quadrotor micro air vehicle. PhD Thesis, Australian National University, Australia.

[9] J. Wang, M. S. Geamanu, A. Cela, H. Mounier, and S. I. Niculescu, "Event driven model free control of quadrotor," in 2013 IEEE International Conference on Control Applications (CCA), 2013, pp. 722-727.

[10] Event Driven Intelligent PID Controllers with Applications to Motion Control." [Online]. Available: http://citeweb.info/20110499270. [Accessed: 27-Dec-2016].

[11] K. J. Åström, C. C. Hang, and P. Persson, "Towards intelligent PID control," Annual Review in Automatic Programming, vol. 15, pp. 53-58, Jan. 1989.

[12] R. Madoński and P. Herman, "Model-free control of a twodimensional system based on uncertainty reconstruction and attenuation," in 2013 Conference on Control and FaultTolerant Systems (SysTol), 2013, pp. 542-547.

[13] L. x Ma, D. n Shi, M. x Chen, and X. q Wang, "Application of intelligent PID control for robot," in 2008 IEEE Conference on Cybernetics and Intelligent Systems, 2008, pp. $455-458$.

[14] S. Bouabdallah, P. Murrieri and R. Siegwart (2004) Design and control of an indoor micro quadrotor. In: proceedings of the IEEE international conference on robotics and automation, pp.4393-4398.

[15] Z. Fang, Z. Zhi, L. Jun, and W. Jian, "Feedback linearization and continuous sliding mode control for a quadrotor UAV," in 2008 27th Chinese Control Conference, 2008, pp. 349353.

[16] X. Zhang, X. Li, K. Wang, and Y. Lu, "A Survey of Modelling and Identification of Quadrotor Robot," Abstract and Applied Analysis, vol. 2014, p. e320526, Oct. 2014.

[17] Y. Bouzid, H. Siguerdidjane, Y. Bestaoui, and M. Zareb, "Energy Based 3D Autopilot for VTOL UAV Under Guidance \&amp; Navigation Constraints," J Intell Robot Syst, pp. 1-22, Nov. 2016, Doi: 10.1007/s10846-016-0441-1

[18] L. Praly, "An asymptotic analysis of the so called intelligent PID controller," Oct-2010. [Online]. Available: https://halmines-paristech.archivesouvertes.fr/file/index/docid/531004/filename/Innocent_P_Co ntroller.pdf. [Accessed: [Accessed: 27-Dec-2016]27-Dec2016].

[19] Y. Bouzid, H. Siguerdidjane and Y. Bestaoui, "Improved 3D trajectory tracking by Nonlinear Internal ModelFeedback linearization control strategy for autonomous systems, " 6th IFAC Symposium on System Structure and Control (SSSC), IFAC-PapersOnLine, vol. 49, no. 9, pp. 1318, Istanbul, Turkey, 22-24 June 2016. 